\title{
Combining 3D technologies for cultural heritage interpretation and entertainment
}

\author{
J.-A. Beraldin ${ }^{\text {a }}$, M. Picard ${ }^{\text {a }}$, S.F. El-Hakim ${ }^{\text {a }}$, G. Godin $^{\text {a }}$, V. Valzano ${ }^{\text {b }}$, A. \\ Bandiera ${ }^{\mathrm{b}}$ \\ $a^{*}$ Institute for Information Technology, National Research Council Canada, \\ Ottawa, ON, Canada \\ $\mathrm{b}^{* \star}$ SIBA Coordination, University of Lecce, Lecce, Italy
}

\begin{abstract}
This paper presents a summary of the 3D modeling work that was accomplished in preparing multimedia products for cultural heritage interpretation and entertainment. The three cases presented are the Byzantine Crypt of Santa Cristina, Apulia, temple C of Selinunte, Sicily, and a bronze sculpture from the $6^{\text {th }}$ century BC found in Ugento, Apulia. The core of the approach is based upon high-resolution photo-realistic texture mapping onto 3D models generated from range images. It is shown that three-dimensional modeling from range imaging is an effective way to present the spatial information for environments and artifacts. Spatial sampling and range measurement uncertainty considerations are addressed by giving the results of a number of tests on different range cameras. The integration of both photogrammetric and CAD modeling complements this approach. Results on a CDROM, a DVD, virtual 3D theatre, holograms, video animations and web pages have been prepared for these projects.
\end{abstract}

Keywords: range camera, photogrammetry, 3D animation, cad, laser scanning, resolution, measurement uncertainty, cultural heritage

\section{INTRODUCTION}

In recent years, high-resolution recording of heritage sites and cultural artifacts (as-built reality) has stimulated a lot of research in computer graphics and vision, and also in photogrammetry. If the only purpose is the generation of photo-realistic images for visualization, then purely image-based rendering techniques offer a general solution. However, if the goal is to analyze the works, to preserve and share a record of their geometry and appearance (digital surrogate), then explicit shape information must be acquired. A 3D model contains a wealth of information that can be analyzed and enhanced ${ }^{1}$. Sites that must be closed for conservation reasons can still be studied and visited. Virtual restoration can be used to improve the understanding of a site (legibility of textual and pictorial information) without turning to interventions often traumatic for the original copy. Elements that were added in a site over the years can be removed and the digital 3D model of that site can then be viewed in the correct historical context. Multimedia CDROMs about the history associated to a particular historical or archaeological site, can use spatial information instead of the usual 2D images that are flat and don't show the three-dimensionality of that site or environment. The core of the approach presented here is based upon high-resolution photorealistic texture mapping onto 3D models generated from range images. Spatial sampling and range measurement uncertainty considerations are addressed by giving the results of a number of

\footnotetext{
* angelo.beraldin@,nrc-cnrc.gc.ca
}

*** siba@siba2.unile.it; 
tests on different range cameras. In choosing the proper resolution, one has to consider the intended application of the work. This application might be visualization in a virtual reality theater for the general public. In this case, the visual acuity ( 1 arc-min) of the average viewer must taken into account $^{2}$. In other cases, 3D documentation aimed at an expert might requires detail levels that go beyond current technology. Here, one must study carefully commercial offerings and compare systems in order to achieve the best possible results within physical, practical and costs limits.

The two sites presented here are aimed at the general public and to a certain degree to the expert. To achieve these goals, the integration of both photogrammetric and CAD modeling was used to complement the approach presented above. It is shown that three-dimensional modeling from range imaging is an effective way to present the spatial information about sites. The first site is the Byzantine Crypt known as the Crypt of Santa Cristina (see Figure 1), which is located in Carpignano (LE), Italy. Results on a CDROM, a DVD, virtual 3-D theatre, holograms and video animations have been prepared for this project.

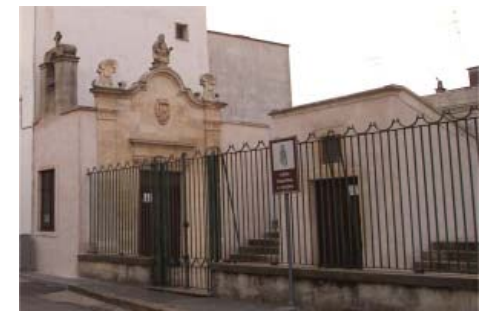

a)

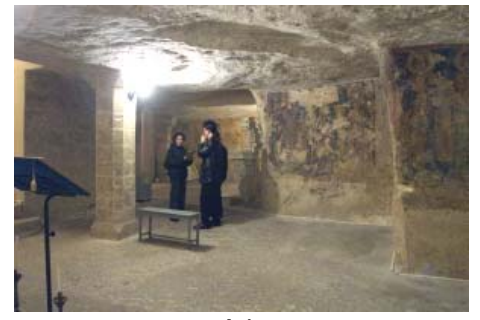

b)

Figure 1. Byzantine Crypt, a) two outside entrances, b) interior located underground.

The second site is Temple $C$ of Selinunte, Sicily. For the acquisition of 3D information, both laser scanning and digital photographs using a calibrated camera were used. Three-dimensional modeling was performed using two different 3D laser scanners, photogrammetry and CAD. At the moment the museum room (Figure 2a) containing artifacts from the site in Selinunte was modeled using a mixture of the above-mentioned technologies and later this year, work will start on building a CAD reconstruction from historical information of temple $C$ of the Acropolis of Selinunte (Figure $2 b)$.

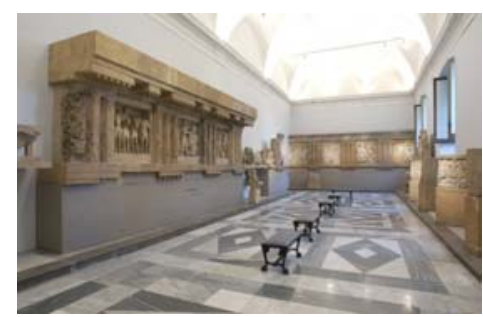

a)

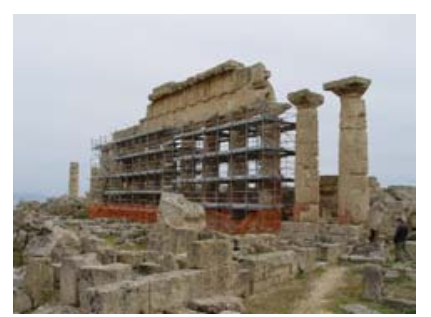

b)

Figure 2. Selinunte project, a) Museum room dedicated to Selinunte, b) Temple C as of 2003 AD.

The third example presented pertains to the modeling of a bronze sculpture known as the Zeus of Ugento, Italy (now part of the collection at the museum of Taranto, Italy). The sculpture measures about $71.5 \mathrm{~cm}$ (height) $\times 45 \mathrm{~cm}$ (hand-to-hand) $\times 18 \mathrm{~cm}$ (back-to-torso). The surface around the head is characterized by fine incisions and decorations. The estimated period is the $6^{\text {th }}$ century BC 
(Figure 3). A high-resolution 3D model (without color) was acquired with a high-resolution laser scanner in order to target the expert user.

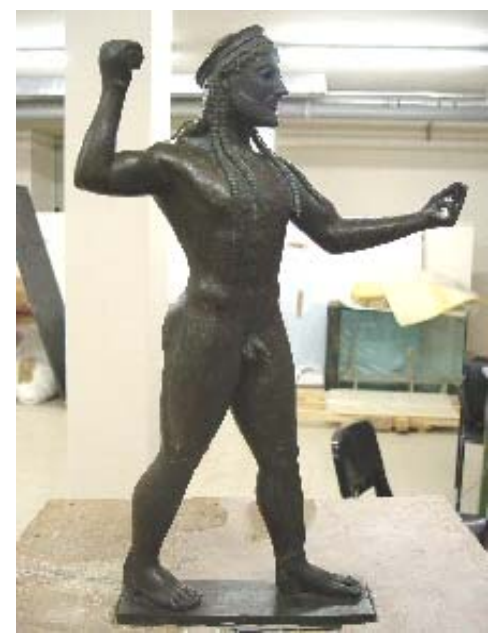

a)

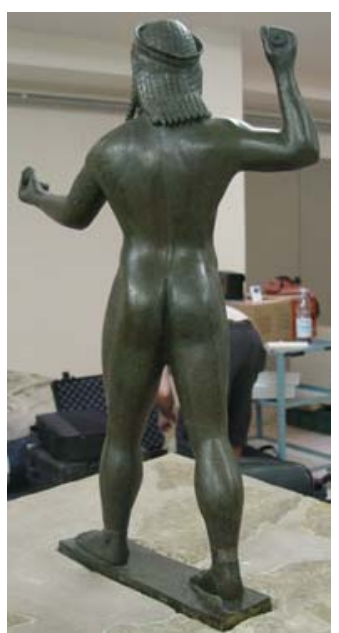

b)

Figure 3. Zeus of Ugento, bronze sculpture $6^{\text {th }} \mathrm{BC}$, Museum of Taranto, Italy, a) height is about 71.5 $\mathrm{cm}, \mathrm{b}$ ) close-up of face.

\section{FUSION OF 3D INFORMATION FOR SITE AND OBJECT MODELING}

When describing and explaining the history of a heritage site or an artifact, the use of spatial information becomes very important in order to facilitate an understanding of that particular site. One can resort to hand drawn or computer generated isometric views, CAD models based on more of less reality and 3D models built from reality. The source of information includes among other things drawing/paintings, papers/digital photographs, or laser scanner data. Some of these provide dimensions directly but others need indirect ways to get scale and/or dimensions.

In many cases, one has to model complex environments. These are composed of several objects with various characteristics and it is essential to combine data from different sensors and information from different sources. There is no single approach that works for all types of environment and at the same time is fully automated and satisfies the requirements of every application $^{3}$. A general approach combines models created from multiple images, single images, range sensors, known shapes, CAD drawings, existing maps, survey data, and GPS data. A survey of the literature on multi-sensor data fusion can generate a long list of papers and books describing the theory and the different applications where data fusion is critical. We will say that it is known that multi-sensor data fusion techniques combine data from multiple sensors and related information from associated databases, to achieve improved accuracies and more specific inferences than could be achieved by the use of a single sensor alone. An example of this approach is the integration of laser scanning and close-range photogrammetry from a multi-sensor and information fusion point of view. Beraldin presents the key features of different laser scanner technologies and photogrammetry-based systems that should be considered in order to realize the benefits expected in a multi-sensor platform ${ }^{2}$. Some examples are given to illustrate the techniques. In particular, two categories of applications are presented by the author, i.e., information augmentation and uncertainty management. Information augmentation refers to a situation where each sensor provides a unique piece of information to an application and fusion extends, for 
example, the system's spatial/temporal coverage. The work on the Byzantine Crypt and Selinunte are examples based on this approach. Uncertainty management is a very important and a critical part of multi-sensor data fusion techniques. It covers situations where different sensors measure the same object/site from different locations or times or even users. In order to deliver the best description of the object/site (lowest uncertainty), one must manage the uncertainties link to the sensing devices, the environment and a priori information (e.g. a particular user). The modeling of the bronze sculpture uses this approach. The objectives of the data fusion are to minimize the impact of those uncertainties and to get the most out of the multi-sensor platform. In other words, one must justify the increased cost and complexity of a multi-sensor solution.

\section{CARPINIANA: A VIRTUALIZED BYZANTINE CRYPT}

\subsection{Modeling techniques used for the Carpiniana project}

We opted to represent the Byzantine Crypt of Santa Cristina (Carpiniana project) using both photogrammetric techniques for the outside (using ShapeCapture software), and, for the inside dense 3D laser scanner information combined with high-resolution color images. Irregularly shaded walls covered with a number of fairly well preserved frescoes made us decide to model the inside with a laser scanner. During the course of history, a Baroque altar was added (1775 AD) along with three pillars that replaced one that collapsed. These elements can all be removed in the 3D model so the site could be viewed in the correct historical context. Many aspects of sensing and modeling must be understood before starting such a large project. The typical processing pipeline used for $3 \mathrm{D}$ modeling includes geometric modeling and appearance modeling ${ }^{4-8}$. Here, we summarize what was done for the Byzantine Crypt. The detailed technical aspects of the project are described in Beraldin et al. [9]. Other virtual heritage projects describe the different steps used for 3D modeling and visualization [4-7].

\subsubsection{Geometric Modeling}

A model is a digital representation of an object or a site on which one can perform operations. Acquiring dense $3 \mathrm{D}$ data of surfaces has been a hot topic of research in the last 20 years ${ }^{10}$. Though not as mature as photography, 3D imaging is seeing new applications emerging every year. Numerous commercial systems are available to measure dense 3D data. The Byzantine Crypt is relatively large $(16.5 \mathrm{~m}$ by $10 \mathrm{~m}$ by $2.5 \mathrm{~m})$ and we wanted to model it with a fairly high spatial resolution. For these kinds of environments, there are not a lot of range cameras on the market that could provide us with the desired level of spatial resolution and low measurement uncertainty ${ }^{10}$. In fact, this range of distances represents the transition between optical triangulation and time of flight technologies.

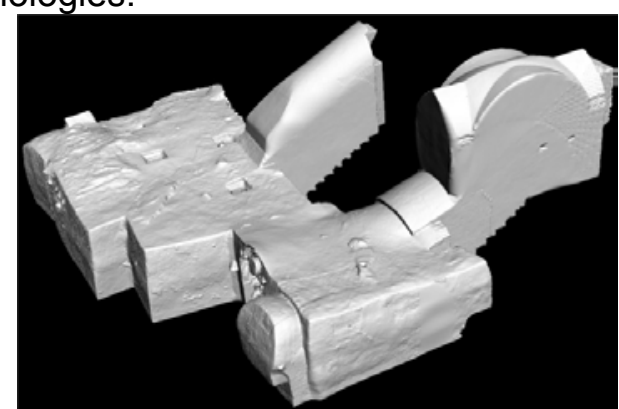

a)

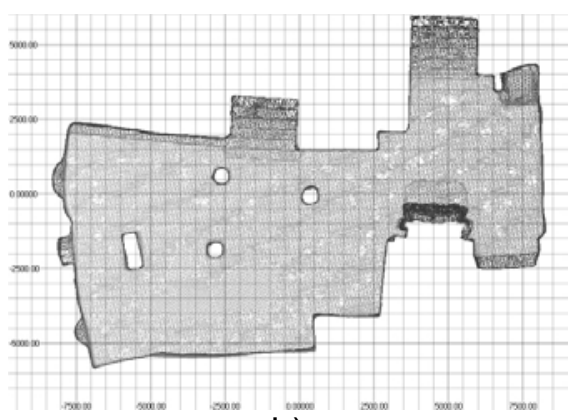

b)

Figure 4. Complete 3D model of the Byzantine Crypt, a) view from outside shown with synthetic shading, b) floor plan generated from an orthographic view of the 3D model. 
In order to create a dense 3D model of the Byzantine Crypt, a MENSI SOISIC-2000 scanner was used. This triangulation-based laser scanner can acquire 3D images at a minimal distance of $0.8 \mathrm{~m}$ and at up to $10 \mathrm{~m}$. The range uncertainty on a cooperative surface at $2.5 \mathrm{~m}$ is about $0.4 \mathrm{~mm}$ (1 sigma) and the data rate is $100-3 \mathrm{D}$ points per second. To make the scanning time manageable, it was decided to use a sampling step on the mesh of $5 \mathrm{~mm}$ (spatial resolution of the model mesh). The range uncertainty after pair-wise 3D image alignment gave about $0.8 \mathrm{~mm}$ (1 sigma) at a distance of $2.5 \mathrm{~m}$. Figure $4 \mathrm{a}$ presents the complete 3D model (without color information) that would appear if one could see through the ground. From this model, a floor plan was created (Figure 4b). A number of models with different levels of complexity were created from the original data using the Polyworks Modeler software from Innovmetric. At the highest quality, the spatial resolution on the wall is about $5 \mathrm{~mm}$ and on the ceiling and floor, $15 \mathrm{~mm}$. As mentioned earlier, the range uncertainty is about $0.8 \mathrm{~mm}$.

Realistic estimate of the time to acquire the range images, build a 3D model and the determination of the required quality of model is very critical for such a project. For this site, with an average scan time on the walls of 80 minutes per 3D image, a total of 92 hours were spent in the Crypt. During that time, fifty 3D images were acquired for the Crypt along with thirty 3D images for the altar. The 3D model was created over a period of one month. The acquisition of the texture took 3 days and the actual mapping was done in 4 days. Figure 5 summarizes the time allocation for the scanning of the site. A large amount of time was dedicated to the actual scans. The second largest slice of time was dedicated to finding the next best view (manually measuring an area on the wall, moving the equipment, pre-scanning an area in order to control the data). Most scanners operate at a rate of 1000 or more 3D samples per second and hence any improvement will come from the reduction of the time to required for finding the next best view ${ }^{11}$.

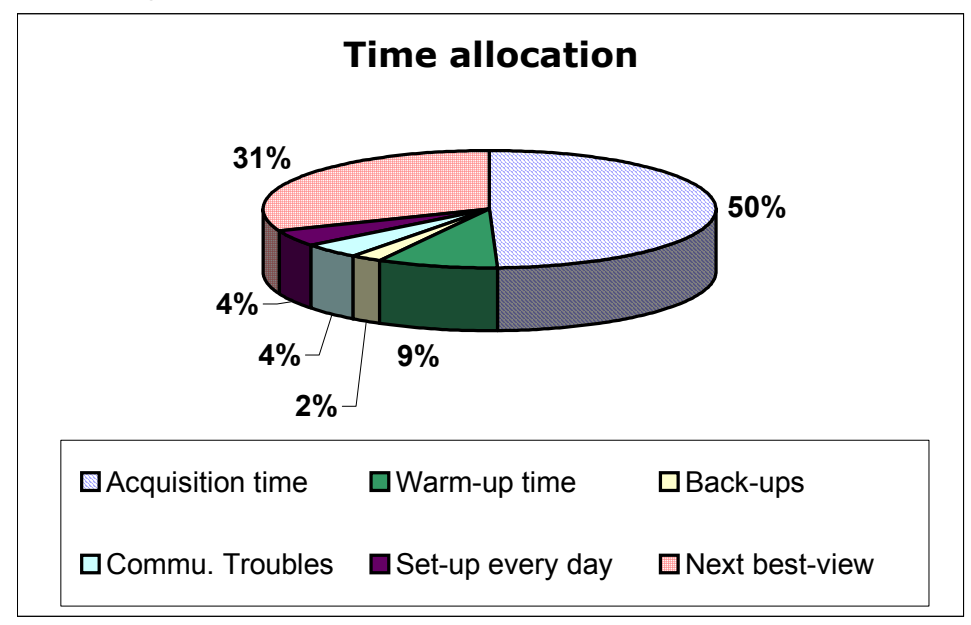

Figure 5. Time allocation for scanning the Byzantine Crypt using a Mensi SOISIC-2000.

\subsubsection{Appearance (Color Texture)}

Appearance modeling includes methods like Image Perspective Techniques (IPT) and Reflectance Modeling (RM). The true appearance of an object is the result of the interaction of light with material. The knowledge of such information is important in order to reproduce hypothetical lighting conditions under varying observation points. For this project, texture mapping using IPT was 
chosen (with TexCapture software). With current CCD and CMOS technology, access to high quality texture images for IPT is now within reach of everyone. Though the Mensi provides 2D images from its internal video camera, the resolution and color quality is not acceptable for our application. So a lens-interchangeable SLR-type 6-MegaPixel digital camera was used for the texture acquisition. Proper texturing of the 3D model requires special lighting fixtures in order to control illumination. Good uniformity of the illumination is essential in order to ease the virtual restoration tasks and improve the visual quality of a textured model.

\subsection{CDROM AND VIDEO ANIMATION: CARPINIANA}

We are currently working with 3 models: one 4.6 million-polygonal un-textured model $(10 \mathrm{~mm}$ resolution) of the complete Crypt, a 12.8 million-polygon fully textured model ( $5 \mathrm{~mm}$ resolution) of one half of the Crypt (contains the two apses), and, a lighter textured model with 0.4 million polygons. These different models were further transformed into orthophotos, isometric crosssections and smaller 3D models in order to navigate through the information on the CDROM. All of these representations are aimed at showing the three-dimensionality of the site that is not visible in a typical visit to the site. A movie called "Carpiniana" showing a fly through of the Byzantine Crypt was also prepared. Snapshots of the CDROM and Video animation are shown on Figure 6 and Figure 7 respectively. When the animation was realized, i.e. year 2002, the computing power was not sufficient to deal with the high-resolution model. The software 3D studio Max helped create the animation. For Carpiniana, the model contained 400000 faces, 1/5 of the maximum texture resolution, 13 lights, 5000 frames at a $720 \times 576$ resolution. Today, the full resolution (shape \& texture) could be used along with a more complex lighting arrangement.

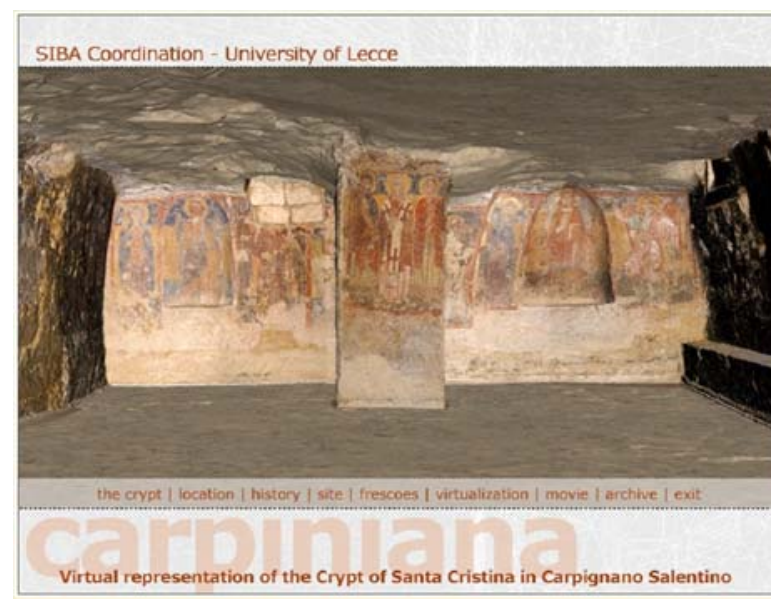

a)

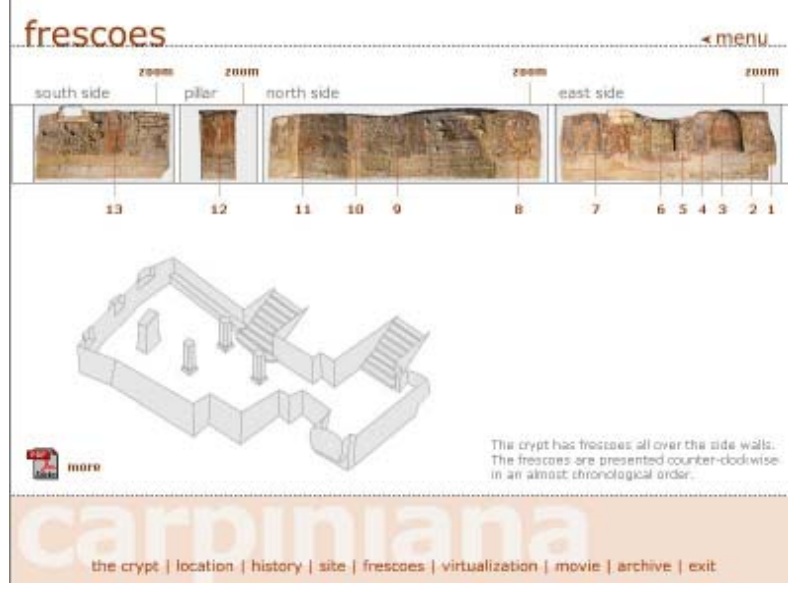

b)

Figure 6. Some screen snapshots for the CDROM CARPINIANA: a) entrance page, b) use of orthophotos generated from $3 \mathrm{D}$ model to navigate through the frescoes.

The presentation of the Byzantine Crypt is now available through a virtual reality theatre (with "il teatro virtuale" software) that can display the full resolution model and allows for a real-time navigation inside the Crypt for further study. A few large format holograms $(1.8 \mathrm{~m} \times 0.85 \mathrm{~m})$ were also produced from the digital 3D model ${ }^{12}$. 


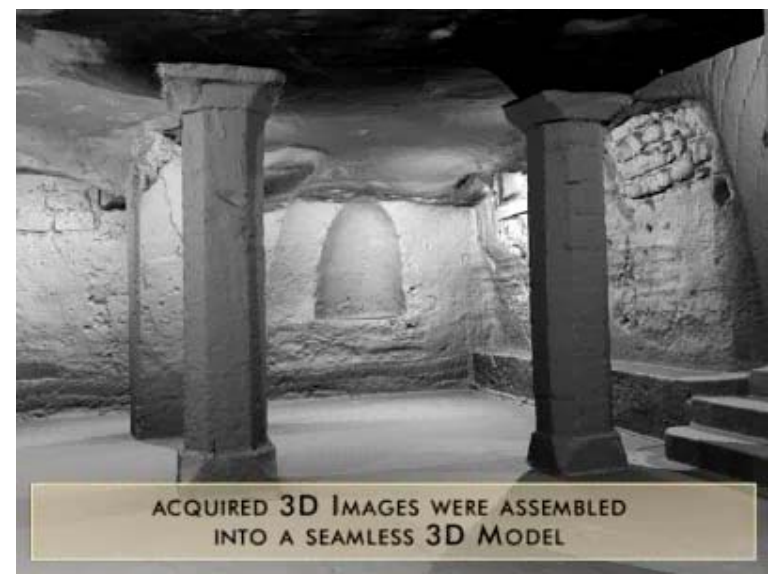

a)

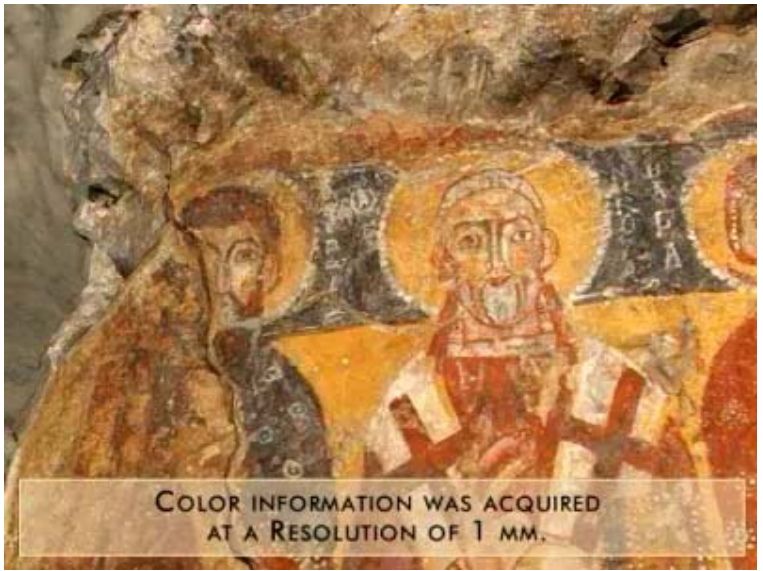

b)

Figure 7. Still images taken from the movie included on the DVD: a) view of crypt without texture, b) view of main pillar with texture.

\section{TEMPLE C OF SELINUNTE (6th century BC)}

The project that started in 2003 is divided into two broad steps, the first step saw the modeling of the frieze of temple $C$ of Selinunte using 3D laser scanning and the second step will see the reconstruction of temple $C$ of the Acropolis of Selinunte using photogrammetry and CAD tools. The 3D model will be based on historical information available at the University of Lecce and at the "Museo Archeologico Regionale" of Palermo, Sicily. The first step saw the scanning and modeling of 3 Metopes from the temple. These are located in the regional museum of archaeology of Palermo, Italy.

\subsection{Practical considerations when using a 3D scanner}

A visit to the museum and to the site allowed the team to plan the activities. One of the main concern was to determine the required spatial resolution and the technical difficulties the team might encounter. Figure 8a shows the aspect of the stone in the triglyph area. The spatial resolution depends on the level of details desired by the project but also by the equipment available and practicality of the choice. The spatial resolution picked for merging the 3D images together determines the size of the smallest triangle on the mesh (Figure $8 b)^{8}$. Other issues can come from the hidden surfaces hard to reach areas and vibrations induced when scaffoldings are used. Both scaffoldings and a mirror placed in the path of the laser beam were used to reach some of those 3D surfaces (Figure 8c).

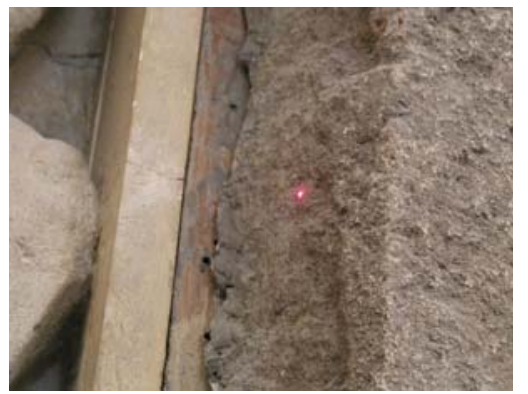

a)

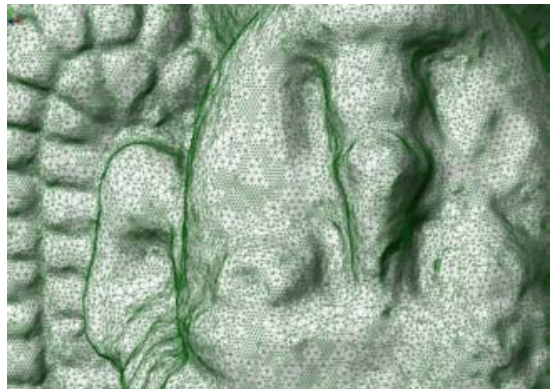

b)

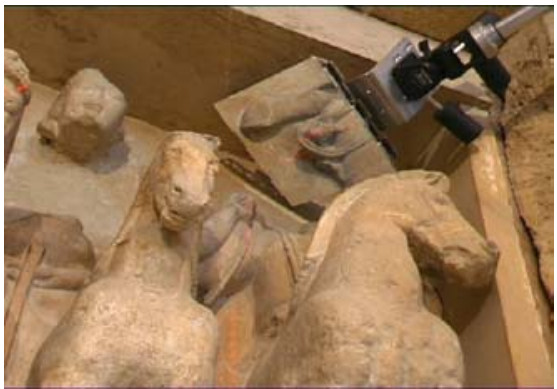

c) 
Figure 8. Practical considerations when using a 3D scanner, a) aspect of the stone in the triglyph area, b) the spatial resolution picked for merging the 3D images together determines the size of the smallest triangle on the mesh, c) use of a mirror to reach hidden 3D surfaces.

Resolution tests were performed using the three lenses supplied with the Minolta 900 . Figure 9 shows the results of those tests. Each lens allows to set the size (resolution) and measurement uncertainty within the 3D volume accessible by the laser scanner. Figure 9a gives the results when the wide-angle lens is used. This lens allows for a large field of view at the expense of a low spatial resolution and high measurement uncertainty. The medium lens (Figure 9b) provides a good compromise between those factors as compare to the lens tele (Figure 9c) which has a field of view that would have required more than $2003 \mathrm{D}$ images as was the case for the lens medium. The mesh resolution for the medium volume lens is about $0.5 \mathrm{~mm}$ and the field of view varies as of function of distance. It is included between $154 \mathrm{~mm} \times 205 \mathrm{~mm}$ at close range and $300 \mathrm{~mm} \times 400$ $\mathrm{mm}$ at the maximum usable range.

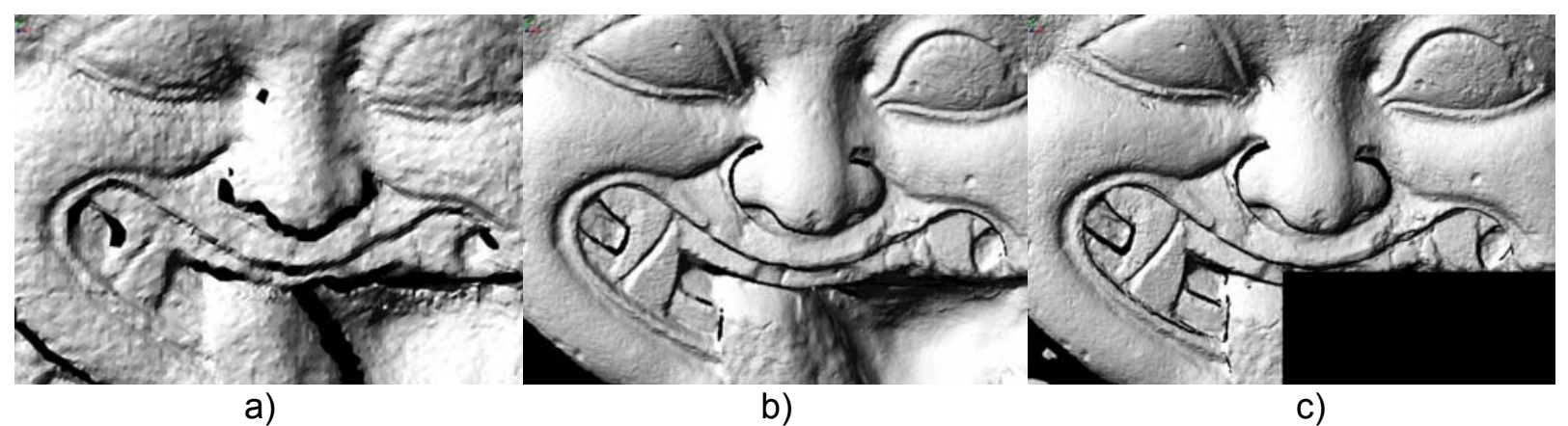

a)

b)

C)

Figure 9. Resolution tests on the Metope Medusa using the Minolta 900, a) wide volume lens, b) medium volume lens (the one that was picked), c) small volume lens.

\subsection{Perseo and Medusa Metope}

Figure 10 shows the results of the modeling of a Metope known as "Perseo and Medusa". Both 3D laser scanning and texture mapping were used to create this model in a manner similar to the project Carpiniana described earlier. The same technique was applied on the other two Metopes associated to temple $C$. The average size of the three Metopes is $1 \mathrm{~m} \times 1 \mathrm{~m} \times 0.4 \mathrm{~m}$ and the number of 3D images required to cover most of the surface is in the order or 175-200 images (depends on the Metope). 


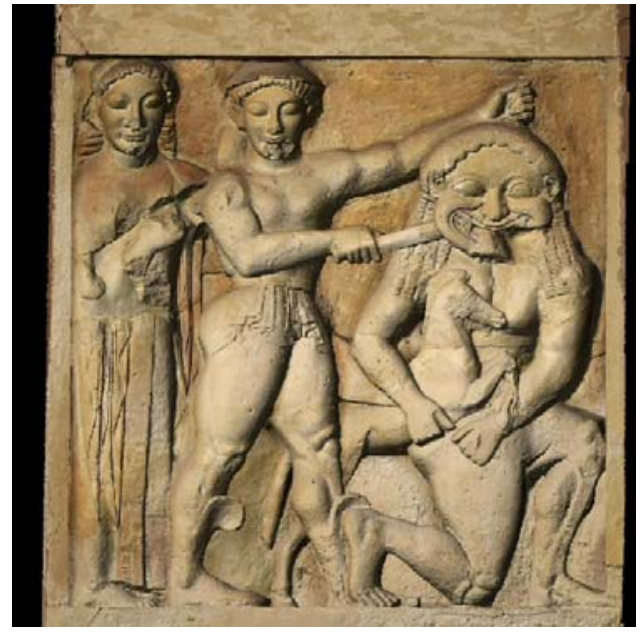

a)

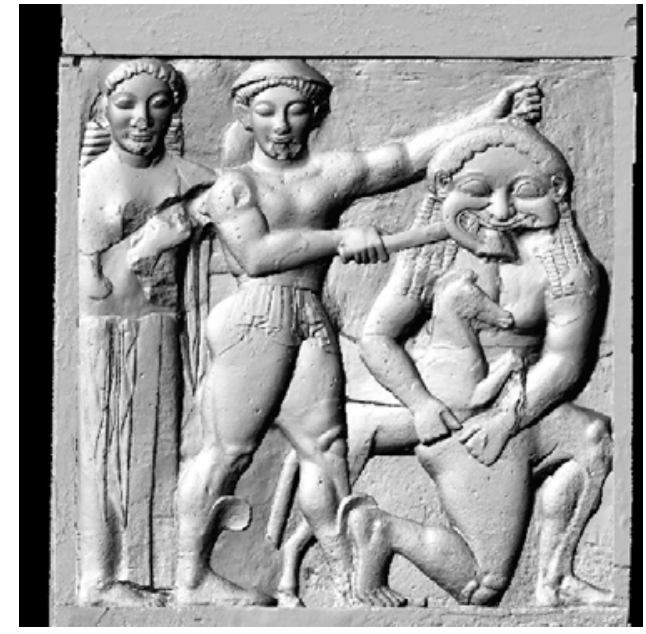

b)

Figure 10. Model of the Metope "Perseo and Medusa", a) texture-mapped 3D model, b) shaded view of the same 3D model.

\subsection{Elements from the frieze of Temple $C$}

The 3D model of the frieze from Temple $C$ including the three Metopes is shown on Figure 11 . We continued the laser scanning work using both the Minolta and Mensi laser scanners on different sections inside the museum room. The first scanner was used to acquire details in the order of 0.5 $\mathrm{mm}$ (see Figure 9b) and the second scanner (Mensi SOISIC-2000) captured details in the range of 2-10 $\mathrm{mm}$. The rest of the 3D model of the room was created using photogrammetry-based modeling techniques and some simple CAD modeling. The 3D model of the frieze generated by laser scanning was registered to the frieze created by photogrammetry-based 3D modeling. A rendering of the complete museum room dedicated to Selinunte is shown on Figure 12. Later this year, the work described above will be integrated with the virtual reconstruction of Temple $\mathrm{C}$.

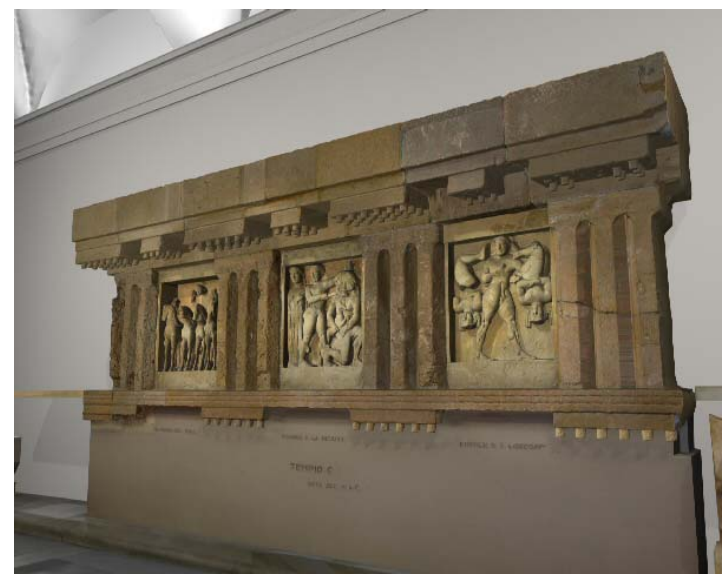

a)

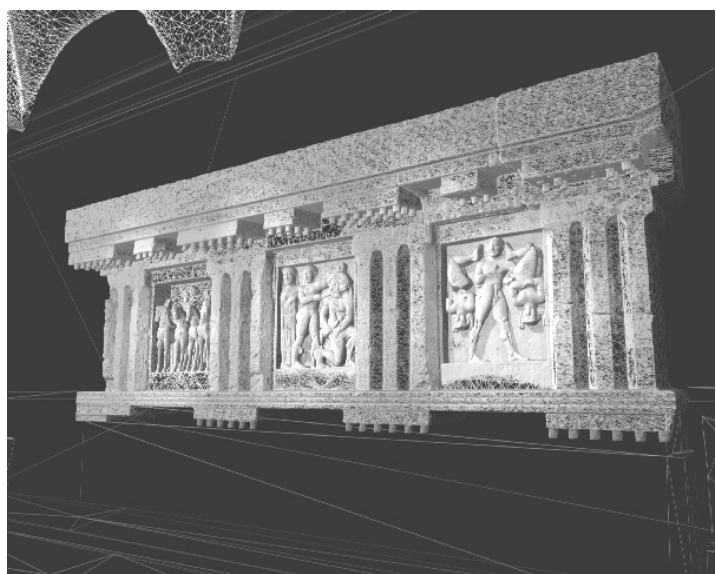

b)

Figure 11. Three-dimensional model of a section of the frieze of Temple $C$ of Selinunte, a) texturemapped 3D model, b) wire-mesh of 3D model showing the levels of details.

Earlier this year, a video animation was realized. The software 3D Studio Max helped create the 3D animation. The model used for this animation contains 5 million faces, the maximum texture 
resolution available, 64 lights, and 6700 frames at a resolution of $720 \times 576$. This represents an important improvement in terms of resolution compared to the animation Carpiniana that was realized two years ago.

\subsection{Museum room in Palermo}

The first part of this project started with the modeling of the frieze of temple C of Selinunte using 3D laser scanning. Later, the second step will see the reconstruction of temple $\mathrm{C}$ of the Acropolis of Selinunte using photogrammetry and CAD tools. While at the museum, it was decided to model the room where the artifacts from different temples from Selinunte are exposed. A rendering of the virtualized room that measures about $21 \mathrm{~m} \times 8.1 \mathrm{~m}$ is shown on Figure 12 . In order to achieve a high degree of realism and accuracy, 3D laser scanning, photogrammetry, CAD and texture processing were used. The quality of the textures was realized using high quality flash lamps, a MacBeth chart and Photoshop. The gray scale available of that chart was used to correct the gamma of the camera and the different levels of illumination. A five-point correction method was used.

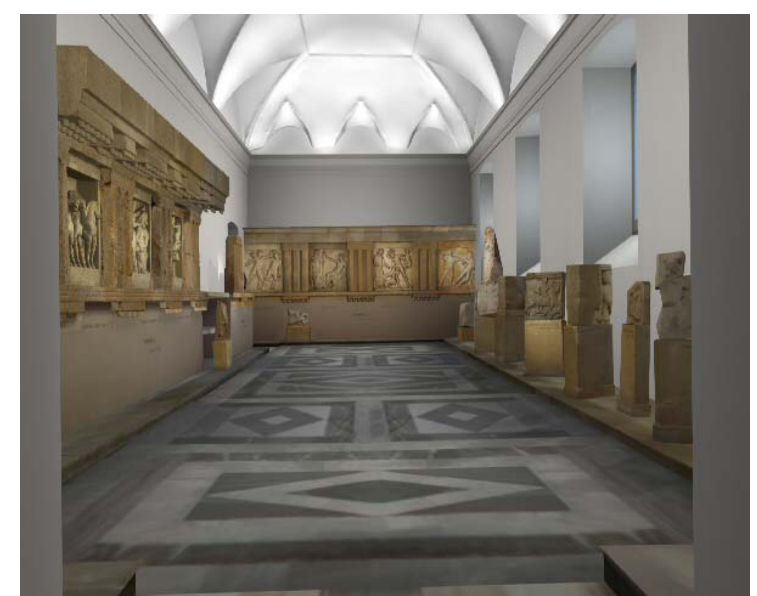

a)

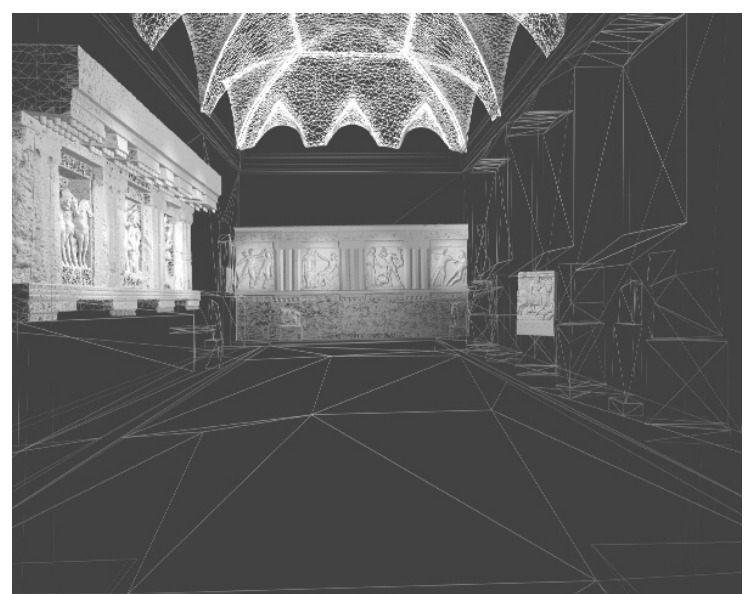

b)

Figure 12. Virtualized Museum room, a) Rendering of the complete 3D model of the museum room dedicated to Selinunte, b) wire-mesh showing the multi-resolution 3D model.

\section{ZEUS OF UGENTO (6th century BC)}

The project stated with the creation of a high-resolution 3D model of the bronze sculpture. Bronze, which is used frequently for sculptures, presents a number of challenges to the 3D photographer. The material is fairly specular even if the sculpture is more than 2500 years old! The surface is dark looking but when a light source is shone on it, both diffuse and specular components appear. Dark surface mean that the signal-to-noise ratio drops and hence the measurement uncertainty goes up. At a specular reflection, the range camera goes in quasi saturation and sometimes in full saturation. When in quasi-saturation, the range data looks good but in reality the uncertainty increase enough to render the model of lesser quality. It was decided to use a laser scanner to model the sculpture as opposed to using a fringe projection system.

The spatial resolution depends on the quality of laser (tight focusing) used in the scanner. A number of tests were conducted to determine the best system to measure the surface of this bronze sculpture. Figure 13 shows the results obtained from the two contenders picked for the 
work. Figure 13a show a photograph of a portion of the face. Figure 13b and c present a shaded view of the partial model of the head for systems Minolta 900-tele lens and ShapeGrabber SG-102.

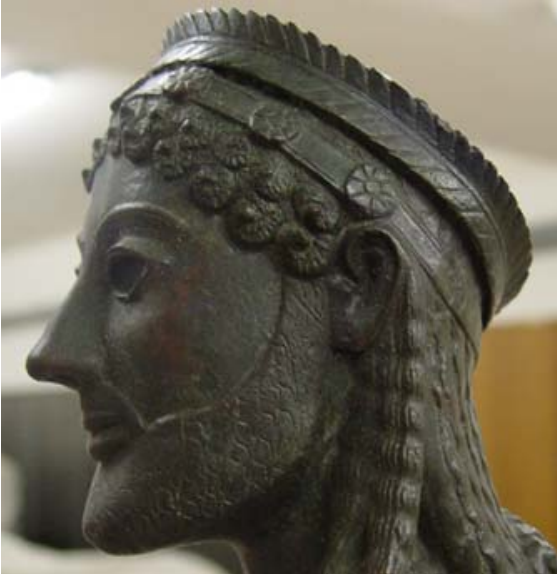

a)

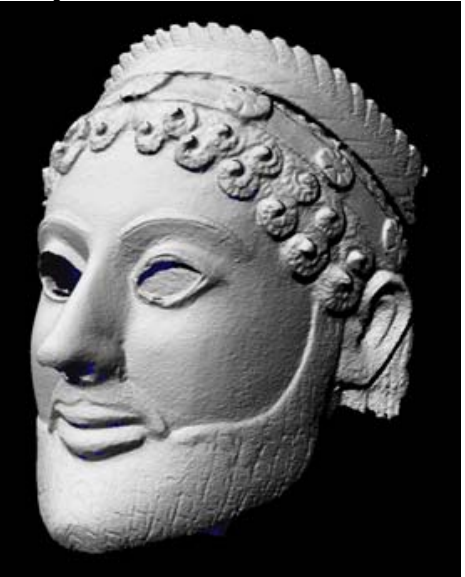

b)

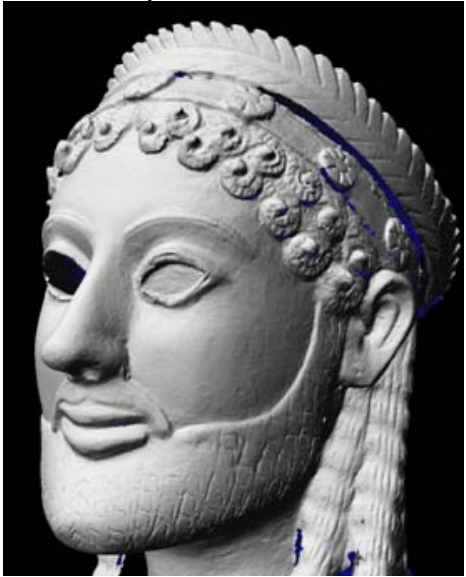

c)

Figure 13. Resolution tests on the sculpture of Zeus. a) photograph, b) shaded view created from 3D data acquired with a Minolta 900 (tele lens) scanner, c) shaded view created from 3D data acquired with a ShapeGrabber SG-102 scanner head.

Below, close-ups show the level of spatial resolution and uncertainty levels for both systems (Figure 14b and c). After a number of tests, it was decide to use the ShapeGrabber SG-102 system to model the sculpture. That system could have been used on the Metope but it was considered a bit cumbersome to use on scaffoldings.

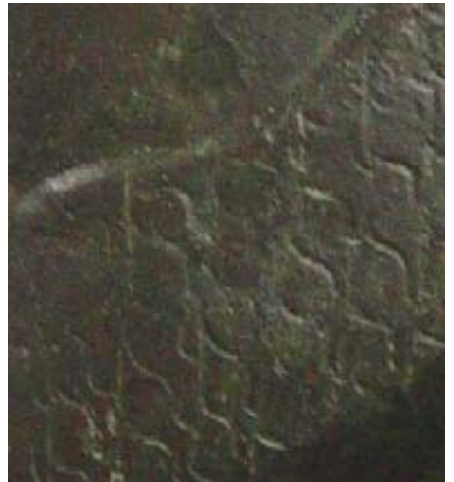

a)

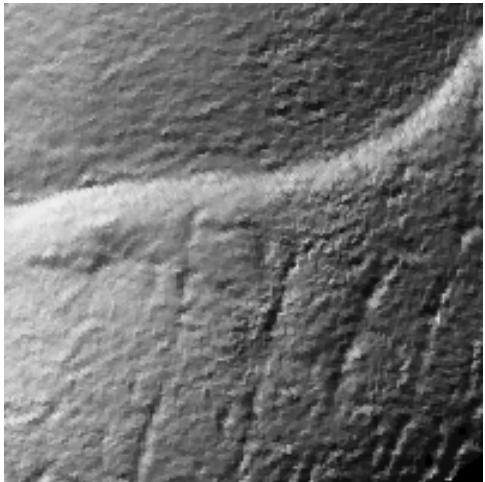

b)

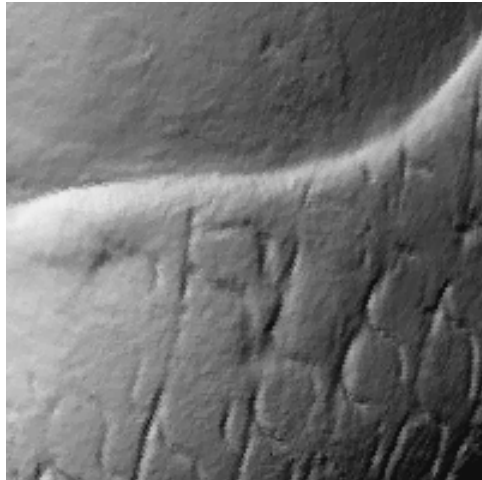

c)

Figure 14. Close-ups, a) a photograph near the left side of the beard area, b) shaded view created from the 3D data acquired with a Minolta 900 (tele-lens) scanner, c) shaded view created from the 3D data acquired with a ShapeGrabber SG-102 scanner head mounted on a translation stage. 


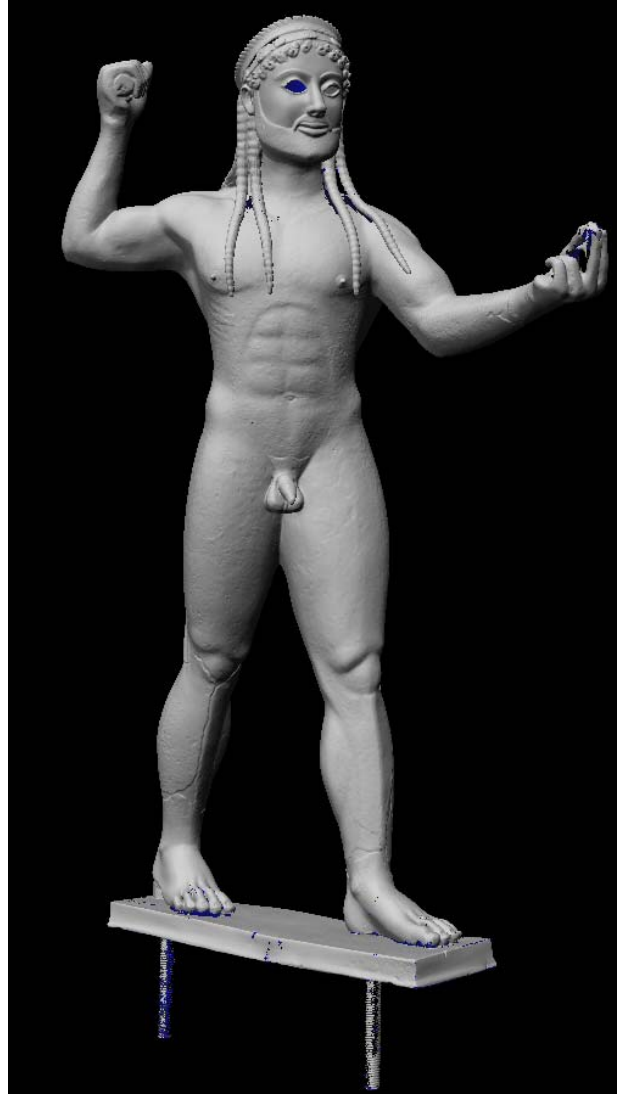

a)

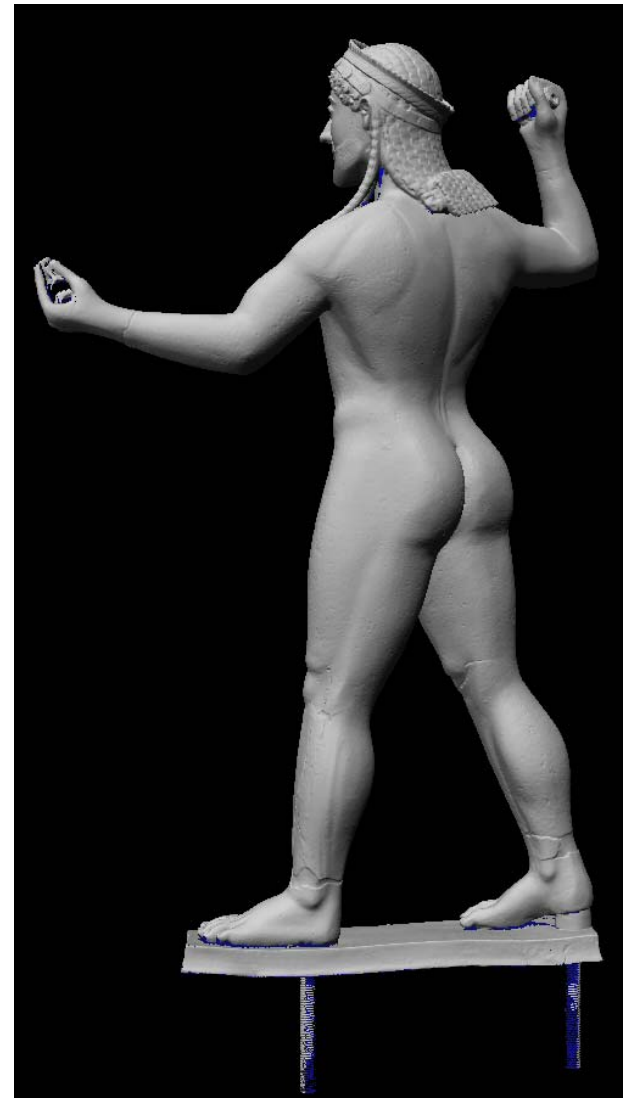

b)

Figure 15. Final 3D model of Zeus of Ugento showed using synthetic shadings (color information has been removed to reveal the surface details), a) frontal view, b) view from the back.

The final model was prepared with about 175 3D images with an average mesh resolution of 0.2 $\mathrm{mm}$. Figure 15 shows two views of the model using synthetic shading. The color has not been acquired for this application. The results of this work will be integrated with historical information already available on this beautiful work of art.

\section{CONCLUSION}

The potential of modeling as-built reality in heritage opens-up applications such as 3D documentation, virtual restoration or as an input to virtualized reality tours. As demonstrated with a Byzantine Crypt, a high degree of realism can be attained by those techniques and the context in which the artifacts were discovered or were used can be recreated. Real world acquisition and modeling is now possible. Technological advances are such that difficulties are more of a logistical nature than technological per se. Models of large objects, structures and environments are possible but as demonstrated here require the combination of a number of techniques. More research work is required to speed up the process of acquisition and modeling. These steps still require a larger amount of time. 
The problem we addressed in this paper is the use of 3D modeling to enhance the understanding of a heritage site that needs to be preserved and shown to more people in order to raise awareness and understanding of the Byzantine and the Greek cultures present in southern Italy. A CDROM, a DVD, a virtual 3D theatre, a number of holograms and two video animations were created to fulfill these hopes. The work on the re-building of temple $C$ of Selinunte is currently underway and will benefit from the experience gained during the work of the Byzantine Crypt of Santa Cristina.

\section{ACKNOWLEDGEMENTS}

The virtualization of the Byzantine Crypt of Santa Cristina was realized within initiative I18 of the "Piano Coordinato delle Università di Catania e Lecce" co-financed by the European Union (FESR, PON 2000-2006). The virtual re-building of temple C of Selinunte, Sicily is part of Activity 4 of the project LandLab (Laboratorio multimediale di ricerca, formazione e comunicazione sui paesaggi archeologici), co-financed by the European Union (PON 2000-2006, Ricerca Scientifica, Sviluppo Tecnologico, Alta Formazione). We would like to thank Gabriele Guidi and Alessandro Spinetti who helped us with some of the laser scans and 3D modeling work. Special thanks go to Andrea Brogi who worked on the 3D animations, Michele Zannoni and Corrado Loschi on the CDROM and DVD, and, Paolo Pulli on the acquisition of the photographs.

\section{REFERENCES}

1. G. Godin, J.-A. Beraldin, J. Taylor, L. Cournoyer, M. Rioux, S.F. El-Hakim, R. Baribeau, F. Blais, P. Boulanger, M. Picard, J. Domey "Active Optical 3D Imaging for Heritage Applications," IEEE Computer Graphics and Applications, 22 (5), pp. 24-36, 2002.

2. J.-A. Beraldin, "Integration of Laser Scanning and Close-range Photogrammetry - the Last Decade and Beyond," Proceedings of the XXth ISPRS congress, Commission VII, Istanbul, Turkey, July 12-23 2004, NRC 46567, pp. 972-983.

3. S.F. El-Hakim, "Three-dimensional modeling of complex environments," Videometrics and Optical Methods for three-dimensional Shape Measurement, San Jose, Jan 20-26, SPIE Vol. 4309, pp. 162-173, 2001.

4. F. Bernardini, I.M. Martin, H. Rushmeier, "High-quality texture reconstruction from multiple scans," IEEE Transactions on Visualization and Computer Graphics 7 (4), pp. 318-332, 2001.

5. S. F. El-Hakim, J.-A. Beraldin, M. Picard, G. Godin, "Detailed 3D Reconstruction of Large-Scale Heritage Sites with Integrated Techniques ," IEEE Computer Graphics and Applications, May/June 2004, 24 (3), pp. 21-29.

6. P. K. Allen, A. Troccoli, B. Smith, S. Murray, I. Stamos and M. Leordeanu, "New Methods for Digital Modeling of Historic Sites," IEEE Computer Graphics and Applications, Nov./Dec. 2003, pp.32-41.

7. J. Stumpfel, C. Tchou, N. Yun, P. Martinez, T. Hawkins, A. Jones, B. Emerson, P. Debevec, "Digital Reunification of the Parthenon and its Sculptures," 4th International Symposium on Virtual Reality, Archaeology and Intelligent Cultural Heritage, Brighton, UK, 2003.

8. M. Soucy, G. Godin, R. Baribeau, F. Blais, M. Rioux, "Sensors and algorithms for the construction of digital 3-D colour models of real objects," Proc. of the IEEE ICIP, V. 2, pp. 409-412, 1996.

9. J-A Beraldin, M. Picard, S.F. El-Hakim, G. Godin, V. Valzano, A. Bandiera, C. Latouche, "Virtualizing a Byzantine Crypt by Combining High-resolution Textures with Laser Scanner 3D Data," VSMM 2002, 25-27 September 2002, Gyeongju, Korea, pp. 3-14. 
10. F. Blais, "A review of 20 years of range sensor development," Journal of Elect. Imaging, 13(1), pp. 231243, 2004.

11. J.-A. Beraldin, L. Cournoyer, M. Rioux, F. Blais, S.F. El-Hakim, G. Godin,, "Object model creation from multiple range images: acquisition, calibration, model building and verification," First Intern. Conf. on Recent Advances in 3-D Digital Imaging and Modeling (3DIM'97), Ottawa, Ont. May 12-15, pp. 326-333, 1997.

12. J.-A. Beraldin, M. Picard, S.F. El-Hakim, G. Godin, E. Paquet, S. Peters, M. Rioux, V. Valzano, A. Bandiera, "Virtual heritage: the cases of the byzantine crypt of Santa Cristina and temple C of Selinunte," VSMM 2004, 17-19 November 2004, Japan. In press. 